\title{
Parametric model order reduction for large-scale systems using Krylov subspace
}

Daming Lou, Siep Weiland

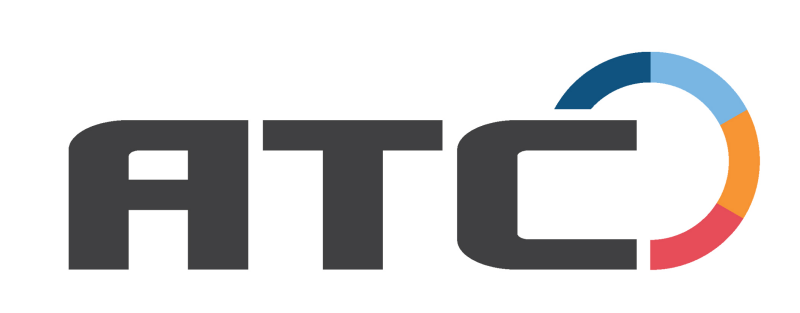

\section{Problem Setup}

Applications Design optimization, parameter estimation, model-based control design, simulation.

Example Parameter calibration

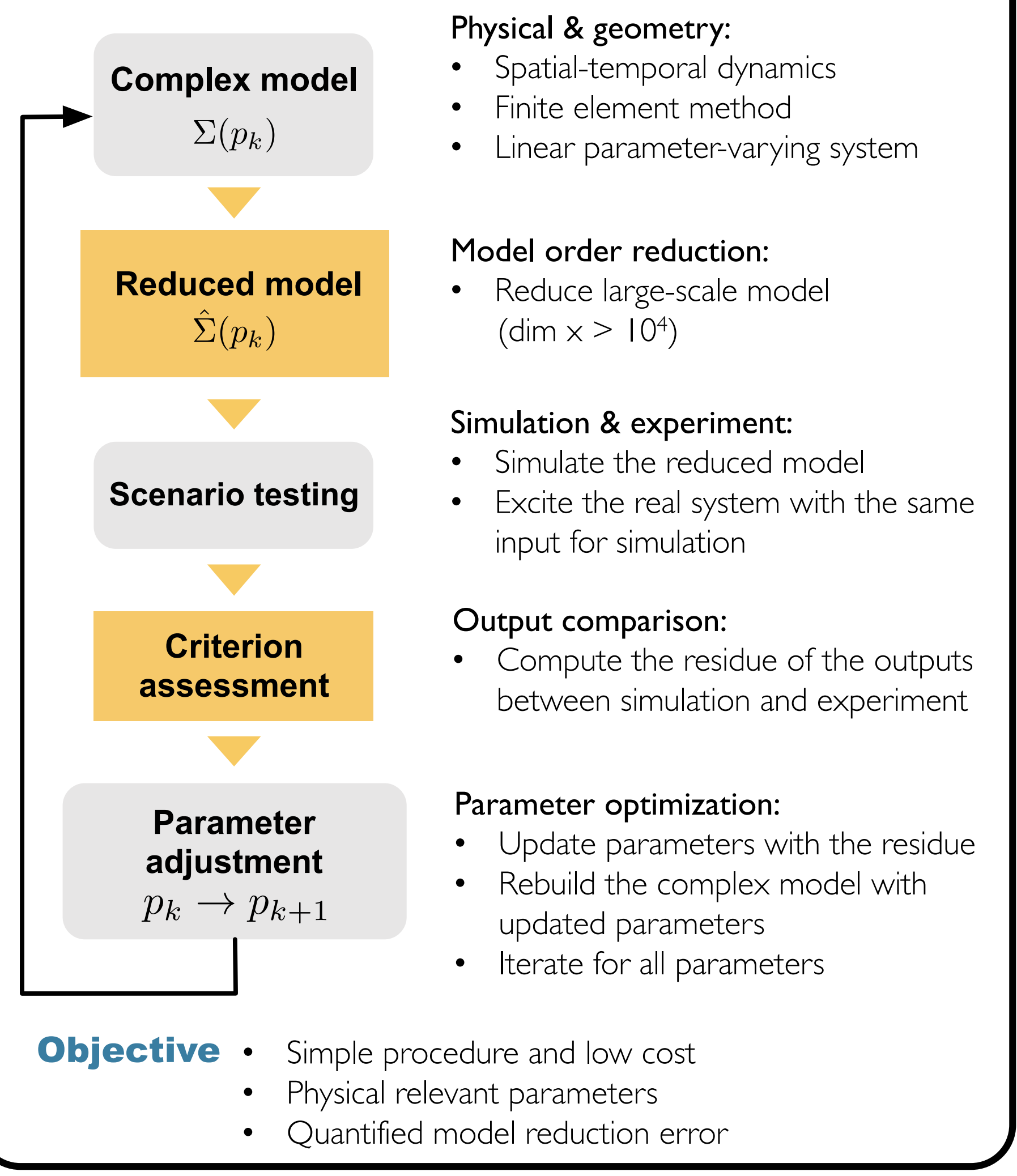

\section{Project statement}

Model order reduction techniques are needed for designs in ultra-high precision systems. Of particular interest is the preservation of the physical relevance of the reduced model.

We present a parametric model order reduction method with explicit error bound.

Multi-frequency and multi-parameter moment-matching

Assumptions

- Parameters are not varying with time

- System matrices are affine functions of parameters.

Laplace variables and physical parameters both expand

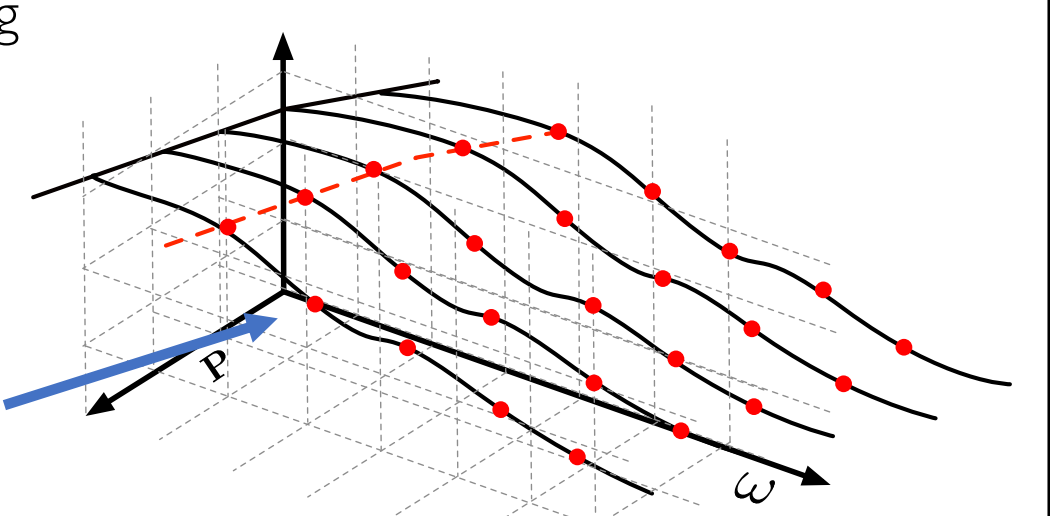

Contribution I

- Generalize moment-matching method and extend to multi-frequency and multi-parameter matching
Thm: Given a system $\Sigma$ of state dimension $n$. Let $\left(s_{0}, p_{0}\right) \in \mathbb{C} \times \mathbb{P}$ and $r<n$. The reduced order system $\Sigma_{r}$ of the state dimension $r$ satisfies

$$
\begin{aligned}
& \eta_{m}\left(s_{0}, p_{0}\right)=\eta_{r, m}\left(s_{0}, p_{0}\right), \quad \text { for }|m|=0, \ldots, M \text {. } \\
& \gamma
\end{aligned}
$$

\section{Error bound}

- Mean value theorem

$$
f(b)=f(a)+R_{n}(b)
$$

- Remainder in Lagrange form

$$
R_{n}(b)=\frac{f^{(n+1)}(\xi)}{(n+1) !}(b-a)^{n+1}
$$
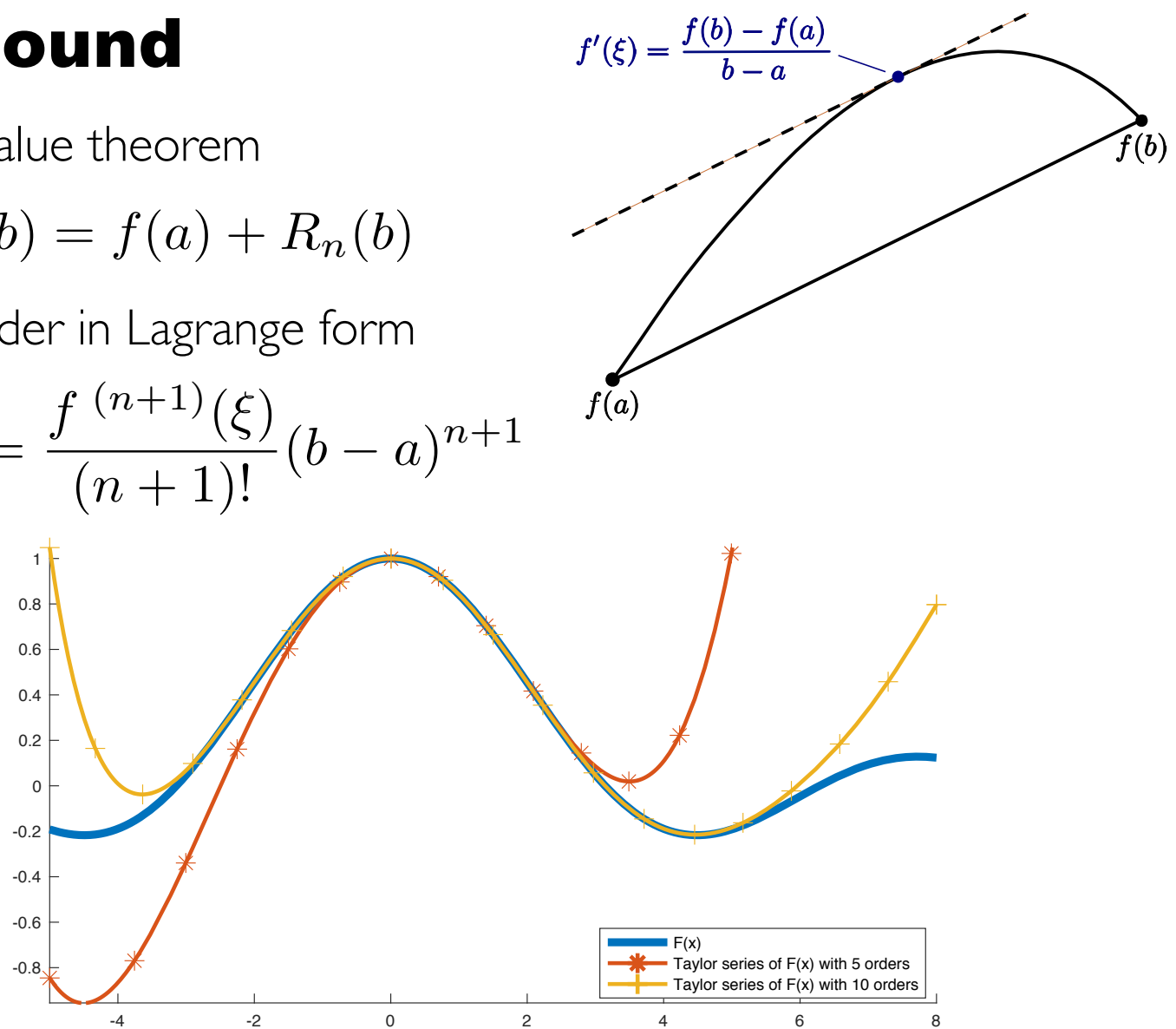

- Error bound

$$
\begin{gathered}
\left\|f(x)-f_{n}(x)\right\| \leq \mathcal{M}|\underbrace{[x-a] \otimes \cdots \otimes[x-a]}_{n+1 \text { copies }}| ; \forall x \in I_{a} \\
\mathcal{M}:=\sup _{\xi \in I_{a}}\left\|\frac{D^{(n+1)} f(\xi)}{(n+1) !}\right\|
\end{gathered}
$$

Prior computed value with the given

reduced order and expansion points

\section{Contribution II}

Prior error bound is proposed and it enables finding optima expansion points and optimal reduced order, iteratively.

\section{A heat transfer case study}

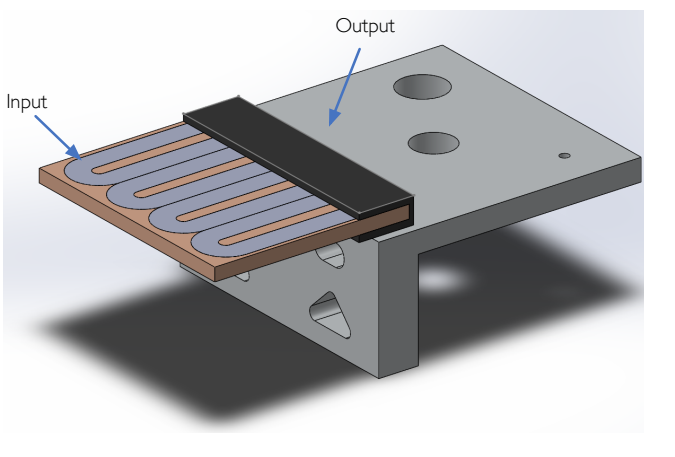

Goal:

Reduce the model while preserving

the heat capacitance parameter Input:

heat generated by the coil Output:

$c_{p} \rho \cdot \dot{T}=\Delta \cdot(\kappa \Delta T)+q \quad$ Temperature at POls

$T(0)=T_{0}$

$\left\{\begin{aligned} E(p) \dot{T} & =A T+B u \\ y & =C T\end{aligned}\right.$

With $\operatorname{dim} T(t) \in \mathbb{R}^{6539}$ and $C_{p}=[200,500,900]$

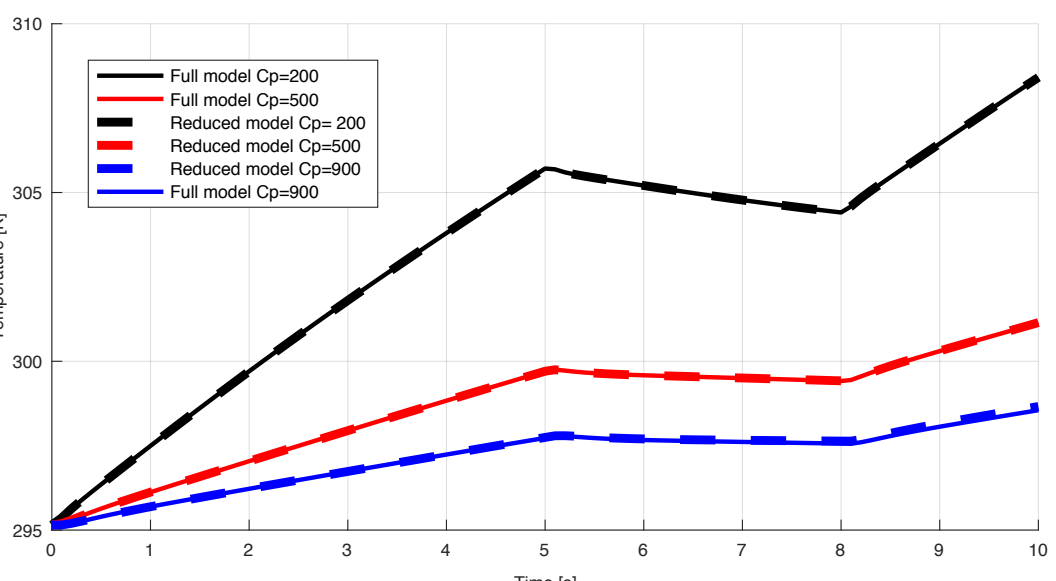

Reduced model: $T_{r}(t) \in \mathbb{R}^{32}$ and $E_{r}(p)=E_{r, 0}+E_{r, 1} C_{p}$

D. Lou and S. Weiland. Parametric model order reduction for large-scale and complex thermal systems. Control Conference (ECC), 2018 European, 20I8 